On the heating mix of ITER

This article has been downloaded from IOPscience. Please scroll down to see the full text article.

2010 Plasma Phys. Control. Fusion 52124044

(http://iopscience.iop.org/0741-3335/52/12/124044)

View the table of contents for this issue, or go to the journal homepage for more

Download details:

IP Address: 128.178.125.176

The article was downloaded on 16/11/2010 at 09:51

Please note that terms and conditions apply. 


\title{
On the heating mix of ITER
}

\author{
F Wagner ${ }^{1}$, A Becoulet ${ }^{2}$, R Budny ${ }^{3}$, V Erckmann ${ }^{1}$, D Farina ${ }^{4}$, \\ G Giruzzi $^{2}$, Y Kamada ${ }^{5}$, A Kaye ${ }^{6}$, F Koechl ${ }^{7}$, K Lackner $^{1}$, \\ N Marushchenko ${ }^{1}$, M Murakami ${ }^{8}$, T Oikawa ${ }^{9}$, V Parail ${ }^{10}$, J M Park $^{8}$, \\ G Ramponi ${ }^{4}$, O Sauter ${ }^{11}$, D Stork ${ }^{10}$, P R Thomas ${ }^{12}$, Q M Tran ${ }^{11}$, \\ D Ward ${ }^{10}, \mathrm{H} \mathrm{Zohm}^{1}$ and C Zucca ${ }^{11}$ \\ ${ }^{1}$ Max-Planck Institut für Plasmaphysik, EURATOM Association, Garching and Greifswald, \\ Germany \\ 2 CEA, IRFM, F-13108 Saint-Paul-lez-Durance, France \\ 3 Princeton Plasma Physics Laboratory, Princeton, NJ 08543, USA \\ ${ }^{4}$ Istituto di Fisica del Plasma, EURATOM- ENEA-CNR Association, 20125 Milano, Italy \\ 5 Japan Atomic Energy Research Institute, Naka Fusion Research Establishment, Naka-machi, \\ Naka-gun, Ibaraki-ken 311-01, Japan \\ ${ }^{6}$ Consultant, Oxford OX12 7HA, UK \\ ${ }^{7}$ Association EURATOM-ÖAW/ATI, Atominstitut, TU Wien, Austria \\ ${ }^{8}$ Oak Ridge National Laboratory, Oak Ridge, Tennessee 37831 \\ ${ }^{9}$ EFDA Close Support Unit, Boltzmannstrasse 2, D-85748 Garching, Germany \\ ${ }^{10}$ Euratom/CCFE Fusion Association, Culham Science Centre, Abingdon, OX14 3DB, UK \\ ${ }^{11}$ Centre de Recherches en Physique des Plasmas, Association Euratom-Confédération Suisse, \\ EPFL, 1015 Lausanne, Switzerland \\ 12 FUSION FOR ENERGY, Joint Undertaking, 08019 Barcelona, Spain \\ E-mail: fritz.wagner@ipp.mpg.de
}

Received 28 June 2010

Published 15 November 2010

Online at stacks.iop.org/PPCF/52/124044

\begin{abstract}
This paper considers the heating mix of ITER for the two main scenarios. Presently, $73 \mathrm{MW}$ of absorbed power are foreseen in the mix 20/33/20 for $\mathrm{ECH}$, NBI and ICH. Given a sufficient edge stability, $Q=10$ - the goal of scenario 2 - can be reached with $40 \mathrm{MW}$ power irrespective of the heating method but depends sensitively inter alia on the H-mode pedestal temperature, the density profile shape and on the characteristics of impurity transport. ICH preferentially heats the ions and would contribute specifically with $\Delta Q<1.5$. The success of the $Q=5$ steady-state scenario 4 with reduced current requires discharges with improved confinement necessitating weakly or strongly reversed shear, $f_{\mathrm{bs}}>0.5$, and strong off-axis current drive (CD). The findings presented here are based on revised CD efficiencies $\gamma$ for ECCD and a detailed benchmark of several CD codes. With ECCD alone, the goals of scenario 4 can hardly be reached. Efficient off-axis CD is only possible with NBI. With beams, inductive discharges with $f_{\mathrm{ni}}>0.8$ can be maintained for $3000 \mathrm{~s}$. The conclusion of this study is that the present heating mix of ITER is appropriate. It provides the necessary actuators to induce in a flexible way the best possible scenarios. The development risks of NBI at $1 \mathrm{MeV}$ can be reduced by operation at $0.85 \mathrm{MeV}$.
\end{abstract}


NBI is a very important option for steady-state operation of DEMO because of its high $\mathrm{CD}$ efficiency. To increase the H-mode accessibility margin, the addition of $20 \mathrm{MW}$ ECH should be considered.

(Some figures in this article are in colour only in the electronic version)

\section{Introduction}

Following a request of the European ITER Domestic Agency, F4E, the heating mix of ITER, the International Thermonuclear Experimental Reactor, was considered by a study group (AG-2) whose magnetic confinement oriented members and their collaborators are authors of this paper (an earlier EU study is summarized in [1]). The study addressed two ITER scenarios considered in the Project Specification [2]: $Q=10^{13}$ at $15 \mathrm{MA}$ with a flat-top time of 300-500 s (operational scenario 2, inductive ELMy H-mode ${ }^{14}$ ) and $Q=5$ at 9 MA under quasi-steady-state conditions (operational scenario 4). Both scenarios are based on H-mode edge conditions. The nominal power thresholds for scenarios 2 and 4 are $70 \mathrm{MW}$ and $40 \mathrm{MW}$, respectively. The $Q=10$ scenario, with $P_{\alpha}=2 P_{\text {ext }}$, is based on core heating and mostly inductive current drive (CD). The $Q=5$ scenario, with $P_{\alpha}=P_{\text {ext }}$, requires both a high bootstrap current $j_{\mathrm{bs}}$ and a CD system with high efficiency $\gamma$ to allow steady-state operation. The demand on the heating system is therefore to produce a plasma state with steep pressure gradients and to globally drive the plasma current with high efficiency.

The heating mix of ITER as given in the ITER Project Specification [2] is $33 \mathrm{MW} \mathrm{NBI}$ (at $1 \mathrm{MeV}), 20 \mathrm{MW}$ of ECH $(170 \mathrm{GHz})$ and $20 \mathrm{MW}$ of ICH $(40-55 \mathrm{MHz})^{15}$. The total power sums up to $73 \mathrm{MW}$ absorbed power. The ITER objectives and the underlying physics are provided by the ITER Physics Base [3] and its recent update [2] and served as a basis for the above-mentioned study and this paper. Here, we analyse the ITER performance with respect to its two major goals- $Q=10$ and $Q=5$, steady state-for different combinations of heating techniques-NBI, ECH and ICH. Specific issues have been whether ICH could be completely abandoned or to which extent NBI could be replaced by ECH. Questions of compatibility of the heating method with fuelling, exhaust or MHD control have not been considered. Various scenarios have been modelled for this study but also already published works of the authors have been included in this paper. Lower hybrid ( $\mathrm{LH})$ heating was not investigated but some results from other works will be quoted to complete the recommendations.

\section{Physics background}

Operational scenario 2 is based on strong core heating in order to access a good H-mode. Heating and $\mathrm{CD}$ during the current rise are needed to minimize flux consumption by achieving a low internal inductance $l_{\mathrm{i}}[4]$. At $l_{\mathrm{i}} \approx 0.8,30 \mathrm{~V} \mathrm{~s}$ will remain for the plateau which will allow $400 \mathrm{~s}$ burn phase. A bootstrap current fraction $f_{\mathrm{bs}}=I_{\mathrm{bs}} / I_{\mathrm{p}} \approx 0.2$ is expected.

Scenario 4 aims at large bootstrap and large externally driven currents. Because of the low CD efficiencies of auxiliary systems, current and density are reduced $\left(I_{\mathrm{p}}=9 \mathrm{MA}\right.$; $n_{\mathrm{e}}=0.7 \times 10^{20} \mathrm{~m}^{-3}$ ). On the other hand, the plasma confinement time in tokamaks increases with $I_{\mathrm{p}}$ and density. In order to offset the performance reduced by the design parameters,

\footnotetext{
${ }^{13} Q$ is the fusion gain defined by the ratio of fusion to external heating power $Q=P_{\text {fus }} / P_{\text {ext }}$.

14 ELMs are MHD instabilities appearing at the edge of the high-confinement $\mathrm{H}$-mode.

${ }^{15} \mathrm{NBI}, \mathrm{ECH}$ and $\mathrm{ICH}$ are the acronyms for neutral beam, electron and ion cyclotron resonance heating.
} 
plasmas with improved confinement $(\mathrm{HH}>1)$ have to be developed ${ }^{16}$. This could be achieved with weak or reverse shear $q$ profiles based on the empirical relation that more strongly reversed shear plasmas attain larger HH factors. Possibly assisted by strong plasma flow a transport barrier develops inside the $q$-minimum, which — together with the one caused by the H-mode edge pedestal (contributing with $\Delta f_{\mathrm{bs}}^{\text {edge }} \approx 20 \%$ to the bootstrap current) — provides the needed level of bootstrap current. As the bootstrap current is generally much larger than the externally driven current, the major goal of a heating method is to serve as actuator providing access to plasmas with good confinement and a high $f_{\mathrm{bs}}$.

These maximum performance plasmas of scenario 4 [5] have large $\beta_{\mathrm{N}}{ }^{17}$ and reside near the operational and stability limits. The stability depends on the pressure peaking factor and the current density profile and has to be improved by additional external measures e.g. to cope with resistive wall modes. As a consequence, there is a strong link between pressure and current density profiles. This is an important difference to scenario 2, where the pressure profile is predicted to have-in comparison-little direct impact on the $q$ profile and overall plasma performance. As steady-state tokamak scenarios will have density, temperature and $q$ profiles, which are a delicate compromise between taking advantage of local transport improvement and staying within an MHD stable operational domain they are not as well defined and predictable as scenario 2. As this study concentrates on the relative merits of different heating mixes in the frame of a defined set of profiles, no detailed analysis has been carried out of the stability properties of the resulting scenarios (see section 4.1).

This report compares the prospects of different heating and CD systems employed in a proper mix for ITER and measured on its objectives. For modelling, transport is taken from the theory-based GLF23 model [6], which can reproduce experimental data well [7]. It specifically reproduces the stiff temperature profiles as experimentally observed. In this case, the edge pedestal in the H-mode plays a dominant role because it governs the plasma temperatures over the whole plasma cross-section.

One has to be aware that major uncertainties are introduced by a lack of predictability of fundamental plasma characteristics. Figure 1 shows the relation between $Q$ and the pedestal temperature for scenario 2 conditions [8]. The external heating is assumed to start at the maximal available power to achieve the H-mode and is then reduced to $47 \mathrm{MW}$ as $\alpha$-power increases. $Q>10$ could only be achieved with $T_{\text {ped }} \geqslant 5.2 \mathrm{keV}$, which could be the upper limit of realistically expected pedestal temperatures. Unfortunately, there is little theoretical understanding and experimental guidance on the critical gradients and the radial extent of the constituents forming the $\mathrm{H}$-mode edge barrier. In particular, the experimental base on pedestal parameters is specifically poor for heating methods alternative to NBI.

Also particle transport, which defines the density profile shape, is crucial. For ITER flat density profiles are assumed. There is, however, strong experimental evidence that a turbulent convective inward flow in the transport physics of scenario 2 might peak the density profile towards low collisionality [9]. As shown here, for peaked $n_{\mathrm{e}}, Q$ would increase by about $\Delta Q>1$ as long as the core impurity concentration remains unchanged.

Depending on the dominant turbulent mode [10] both tokamaks [11] and stellarators [12] often show flat density profiles in EC-heated plasmas at low collisionality. This feature would be less favourable for high $Q$ in ITER. In present experiments, increasing electron heating has a significant effect on $T_{\mathrm{e}} / T_{\mathrm{i}}$ and tends to increase the trapped-electron-mode (TEM) contribution. In ITER, however, even at low collisionality and strong electron heating, $T_{\mathrm{e}}$ will be close to $T_{\mathrm{i}}$ and it is not expected that TEM will be dominant. This is why ITER is expected to have a

\footnotetext{
16 The factor HH represents the ratio of $\tau_{E}$ to that of the ITER 98( $y$,2) ELMy H-mode scaling.

$17 \beta_{\mathrm{N}}=\beta a B / I_{\mathrm{p}}$, with $\beta=$ volume averaged pressure/magnetic pressure.
} 


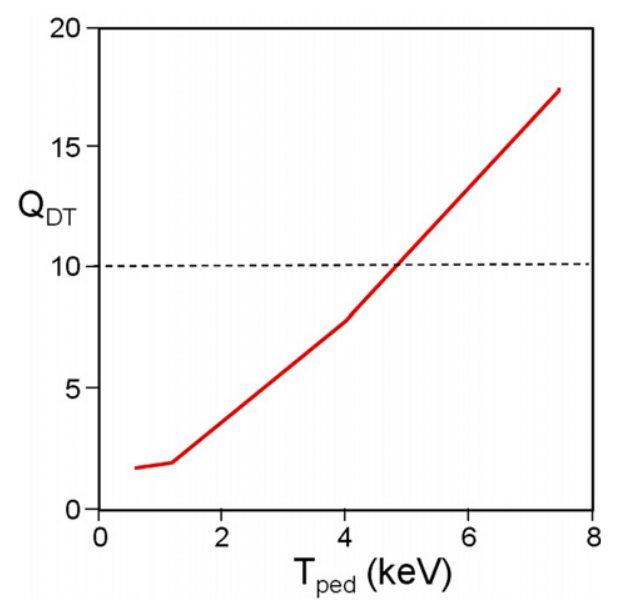

Figure 1. $Q_{\mathrm{DT}}$ versus the pedestal temperature $T_{\text {ped }}$ for the ITER scenario 2 .

peaked density profile [11]. In addition, if the stationary scenario is dominated by ITG modes, increasing electron heating might even slightly increase the density peaking [10]. Because of the uncertainties of turbulent particle transport, in the simulations both flat and peaked density profiles were considered.

The impurity concentration depends on edge sources and for He on the central one and on the diffusive and convective transport, which could be inward directed. He transport ( $D_{\text {ash }}$ and $V_{\text {ash }}$ ) is therefore crucial for high fusion yield. Sensitivity studies have been done in [8].

Only ITER can demonstrate the self-organized plasma characteristics with strong central self-heating by fusion $\alpha$-particles. In borderline cases, the $\Delta Q$ increment from direct ion heating (as provided by ICH: $\Delta Q<1.5$ ) could play a role as well as the beam-target fusion interaction as provided by NBI $(\Delta Q \leqslant 0.5)$. Also the impact of sheared rotation as induced by NBI might be beneficial for the creation of an internal transport barrier, ITB, and improved confinement and stability. As this study concentrates on well-defined target scenarios, no further exploration was made of the possible benefits of plasma rotation on scenario development.

\section{CD modelling}

\subsection{Electron cyclotron current drive (ECCD)}

For an accurate calculation of the CD efficiency $\gamma=I_{\mathrm{CD}} n_{\mathrm{e}} R / P_{\mathrm{CD}}$, parallel momentum conservation in the collision processes has to be ensured [13]. The two momentum conserving codes (CQL-3D (Fokker-Planck) [14] and TRAVIS (adjoint technique) [15]) showed consistency in the benchmark. They yield a higher CD efficiency (for typical ITER applications by $\approx 20 \%$ ) in comparison with the-at the time of writing-non-conserving approaches (GRAY [16], TORBEAM [17]).

$\mathrm{ECH}$ has excellent localization of CD power, which is exploited for MHD stabilization. For this purpose, dedicated launchers are installed [18]. The ECCD efficiency is larger in the plasma core but drops, however, strongly towards the plasma edge mainly because of the rising fraction of trapped particles and the drop in $T_{\mathrm{e}}$; see figure 2 . 


\subsection{Neutral beam current drive (NBCD)}

NBI can drive strong global current arising from the fact that due to $Z_{\text {eff }} \neq 1$ and toroidal trapping, the electrons cannot perfectly shield the fast injected ion current. The calculation of the driven current requires the exact description of the ion birth profile due to ionization and charge exchange and that of the slowing down process. For calculating the fast ion current, multi-step ionization processes are taken into account [19]. Orbit effects are important to describe correctly the location of the driven current. It is therefore important that the magnetic equilibrium is accurately provided to account for the large orbits of the fast ions.

The correct description of the electron shielding requires also precise equilibrium description to get the correct trapped electron fraction. For the beam codes used and benchmarked in this study (OFMC [20] and NUBEAM [21]) differences of about 25\% in the $\mathrm{CD}$ efficiency appeared coming mainly from the fast ion current whereas the electron shielding agrees rather well. OFMC calculates an NBI driven current of 2.58 MA and NUBEAM of 2.13 MA, respectively. As the reason for the remaining discrepancies is presently not known both NUBEAM and OFMC were used in parallel and the discrepancies were considered as uncertainties.

\subsection{Summary on CD modelling}

A comparison of CD efficiencies between NBCD and ECCD depends strongly on the conditions prescribed: while for ITER scenario 2, the central ECCD and NBCD efficiencies are comparable, the off-axis ECCD efficiency is substantially lower than that for NBCD, whichcontrary to ECCD_increases with the fraction of trapped particles. This reduction of ECCD necessitates the use of off-axis CD by NBI in scenario 4 (see figure 2).

The physics base for ECCD is quite mature and codes using a fully relativistic momentum conserving approach should give a realistic estimate for the CD capabilities in ITER. The physics base for NBCD is slightly less developed, with some $25 \%$ variation on the modelling side and an unknown fast ion transport that especially affects off-axis CD [22]. The anomalous diffusion of the fast ions is difficult to quantify but should not lead to large discrepancies for central NBCD. Modelling results from present state-of-the-art codes should therefore be regarded as an upper limit, with the total current at on-axis application being the quantity with the smallest uncertainty.

\section{Modelling results}

\subsection{Scenario 2}

Scenario 2 plasma cases were simulated with JETTO [23]. The theory-based GLF23 transport model [6], which is used inside the edge barrier, predicts that the plasma density should have a peak in collisionless ITER plasmas. Therefore both imposed flat and self-consistent peaked density profiles are used in the modelling thus defining the performance limits given by the density profile shape. It is assumed in all simulations that transport between top of the edge barrier and the separatrix is reduced to the level which keeps normalized pressure gradient $\alpha$ close to but below the critical level $\alpha_{\mathrm{cr}}$. A width of the edge barrier $\Delta=6 \mathrm{~cm}$ at the outer midplane was assumed and kept the same for all cases. $\alpha_{\mathrm{cr}}$ was determined from the linear MHD stability codes MISHKA [24] and ELITE [25] and is found to be limited to $p_{\text {ped }}<130 \mathrm{kPa}$. The limit is indicated in figure 3 by the vertical line.

Figure 3 summarizes the main results of a generic plasma performance study addressing the specificies of the three heating methods and demonstrating the impact of the density profile. 


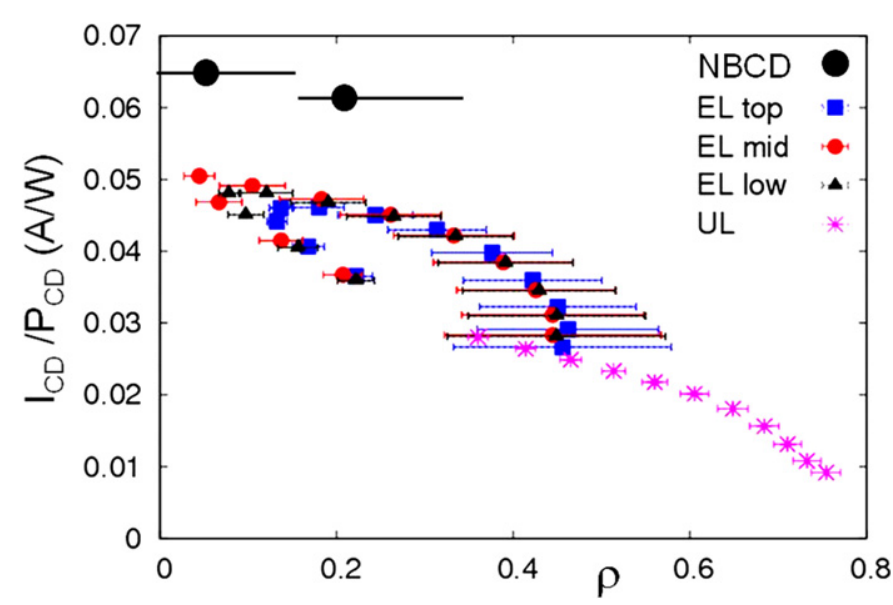

Figure 2. Driven current $I_{\mathrm{CD}}$ to $\mathrm{CD}$ power $P_{\mathrm{CD}}$ versus normalized radius for the ITER $Q=5$ steady-state scenario (scenario 4) for NBCD and ECCD utilizing the ECH injection options (EL: equatorial launcher; UL: upper launcher). The ECCD calculations are done with momentum conservation (TRAVIS [15]). The horizontal bars indicate the width of the deposition profiles. No discrimination is made between high-field and low-field-side absorption; therefore, some curves are double valued.

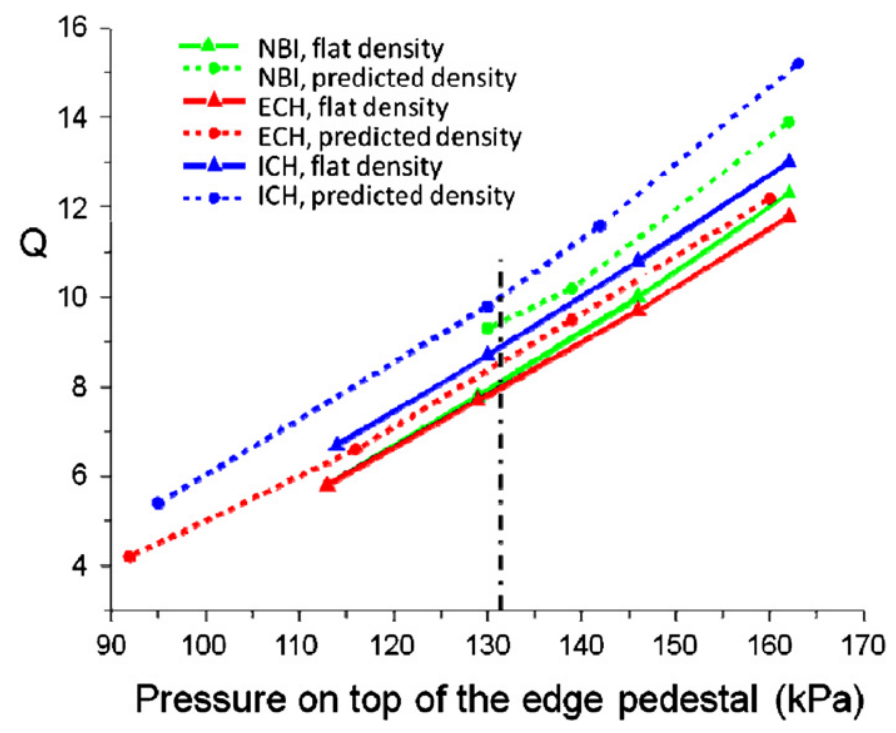

Figure 3. Fusion gain $Q$ for $40 \mathrm{MW}$ of either pure ICH heating (blue lines), pure NNBI with $1 \mathrm{MeV}$ ions (green lines) and pure $\mathrm{ECH}$ (red lines) as a function of thermal plasma pressure on top of the pedestal. Solid lines correspond to simulations with flat density profiles and dashed lines to peaked densities. The dashed-dotted vertical line is the expected edge pressure stability limit. $6 \mathrm{~cm}$ is taken for the barrier width.

The absorbed power is $40 \mathrm{MW}$ for each case, which is the power level sustaining, along with the $\alpha$-particle heating, a $Q=10$ plasma. Peaked density profiles lead to a higher level of fusion gain $Q$ than flat ones with the difference $\Delta Q>1$ for the case of pure ICH. There is a distinct difference in fusion gain between predominantly ion heating and pure electron 
Table 1. Summary of the thermal $\beta_{\mathrm{N}}$ values for the cases considered.

\begin{tabular}{ll}
\hline Scenario & $\beta_{\mathrm{N}}$ \\
\hline NBI, flat density & 1.44 \\
NBI, predicted density & 1.52 \\
ECH, flat density & 1.49 \\
ECH, predicted density & 1.49 \\
ICH, flat density & 1.48 \\
ICH, predicted density & 1.52 \\
\hline
\end{tabular}

heating with $\Delta Q$ reaching $\Delta Q_{\max }<1.5$ between $\mathrm{ICH}$ and $\mathrm{ECH}$ heating. The difference in performance between the reference mix and pure electron heating amounts to $\Delta Q_{\max }<1.0$.

Table 1 shows the thermal $\beta_{\mathrm{N}}$ values for the cases considered here. The values turn out to be below the reference $\beta_{\mathrm{N}}$ of ITER, $\beta_{\mathrm{N}, \text { ITER }}=1.8$ (total $\beta$ !). These discharges are not subject to the ideal limit and neoclassical tearing modes do not depend on $\beta_{\mathrm{N}}$. The most prominent ITER objective depends, however, critically on MHD stability in the plasma periphery with rather involved physics but little predictive understanding. With all reservations it is predicted that ITER may reach $Q \approx 10$ marginally. Therefore, the proper choice of heating mix is of crucial importance. To stress this is the purpose of this exercise. ITER has better chances to reach its $Q=10$ goal with those heating mixes which maximize ion heating (ICH). Scenarios with improved bulk confinement, not considered here, may ease access to $Q=10$ if accessible to ITER.

Scenario 2 flat-top time has recently been analysed in detail for different pedestal temperatures and Ejima parameters [26]. In this study here, the reference heating mix was compared with pure ECCD for the volt-second consumption. Both simulations were done under identical assumptions-flat electron density, same $\alpha_{\text {crit }}$. The following conclusions can be drawn: NBI and ECCD have similar CD efficiencies (though different profiles). Both methods $\left(P_{\mathrm{NBI}}=33 \mathrm{MW} ; P_{\mathrm{ECH}}=40 \mathrm{MW}\right)$ generate plasmas with a bootstrap current of $I_{\mathrm{bs}}=3 \mathrm{MA}$, which is about three times the driven current. Both ECCD and NBCD reduce insignificantly resistive flux consumption (by about $1 \mathrm{~V}$ s per $100 \mathrm{~s}$ of burn) and are not expected to play a big role in extending the burn period of scenario 2 . Total resistive flux consumption for the burn phase of scenario 2 requires approximately $30 \mathrm{~V} \mathrm{~s}$ for $400 \mathrm{~s}$ burn, which is marginally consistent with the capacity of the ITER PF system.

In summary, the exact heating mix is found to be not critical for scenario 2 and could be NBI, ICH as well as ECH. In any case, ICH should be included to utilize the benefits of direct ion heating.

\subsection{Scenario 4}

Unlike scenario 2, where the CD characteristics of the heating methods do not critically enter, scenario 4 was analysed with the help of four distinctly different heating mixes. Heating scenario A represents the baseline ITER mix. All considered cases A-D sum up into $73 \mathrm{MW}$ absorbed power. Table 2 lists the power mix and the CD signatures of each case.

4.2.1. Global considerations. A relation between $Q$ and the bootstrap fraction $f_{\mathrm{bs}}$ can be easily derived using the definitions of $Q=P_{\text {fus }} /\left(P_{\text {heat }}+P_{\mathrm{cd}}\right),\langle\gamma\rangle$ and $f_{\mathrm{bs}}$, respectively. $P_{\text {heat }}$ is an additional heating power associated with $\mathrm{ICH}$ for the heating cases $\mathrm{A}$ and $\mathrm{B}$, which does not contribute to drive current. For fixed $Q, f_{\mathrm{bs}}$ can be expressed as a function of $\langle\gamma\rangle$. For $Q \approx 5, f_{\mathrm{bs}}$ is plotted against $\langle\gamma\rangle$ in figure 4 for the two cases $P_{\text {heat }}=0$ and $P_{\text {heat }}=20 \mathrm{MW}$. 
Table 2. $\mathrm{CD}$ efficiencies $\gamma\left[10^{20} \mathrm{~m}^{-2} \mathrm{~A} \mathrm{~W}^{-1}\right]$, driven currents $I_{\mathrm{CD}}$, the assumed global $\mathrm{CD}$ efficiency $\langle\gamma\rangle$ and the global CD fraction $f_{\mathrm{CD}}$ for the heating scenarios A-D for ITER scenario 4 .

\begin{tabular}{|c|c|c|c|c|c|c|c|c|c|c|}
\hline \multirow[b]{2}{*}{$\begin{array}{l}\text { Heating } \\
\text { scenario }\end{array}$} & \multicolumn{4}{|c|}{ ECCD } & \multicolumn{3}{|c|}{ NBCD } & \multirow{2}{*}{$\begin{array}{l}\mathrm{ICH} \\
P \\
(\mathrm{MW})\end{array}$} & \multicolumn{2}{|c|}{ Global } \\
\hline & $\begin{array}{l}P \\
(\mathrm{MW})\end{array}$ & $\gamma_{\mathrm{CD}}(0)$ & $\gamma_{\mathrm{CD}}(0.4)$ & $\begin{array}{l}I_{\mathrm{CD}} \\
(\mathrm{MA})\end{array}$ & $\begin{array}{l}P \\
(\mathrm{MW})\end{array}$ & $\gamma_{\mathrm{CD}}$ & $\begin{array}{l}I_{\mathrm{CD}} \\
(\mathrm{MA})\end{array}$ & & $\left\langle\gamma_{\mathrm{CD}}\right\rangle$ & $f_{\mathrm{CD}}$ \\
\hline $\mathrm{A} / 20 / 33 / 20$ & 20 & & 0.16 & 0.7 & 33 & 0.3 & 2.3 & 20 & 0.25 & 0.34 \\
\hline $\mathrm{B} / 53 / 0 / 20$ & 53 & 0.22 & 0.16 & 2.4 & 0 & & 0 & 20 & 0.2 & 0.27 \\
\hline $\mathrm{C} / 73 / 0 / 0$ & 73 & 0.22 & 0.16 & 3.4 & 0 & & 0 & 0 & 0.2 & 0.38 \\
\hline $\mathrm{D} / 40 / 33 / 0$ & 40 & 0.22 & 0.16 & 1.7 & 33 & 0.3 & 2.3 & 0 & 0.23 & 0.45 \\
\hline
\end{tabular}

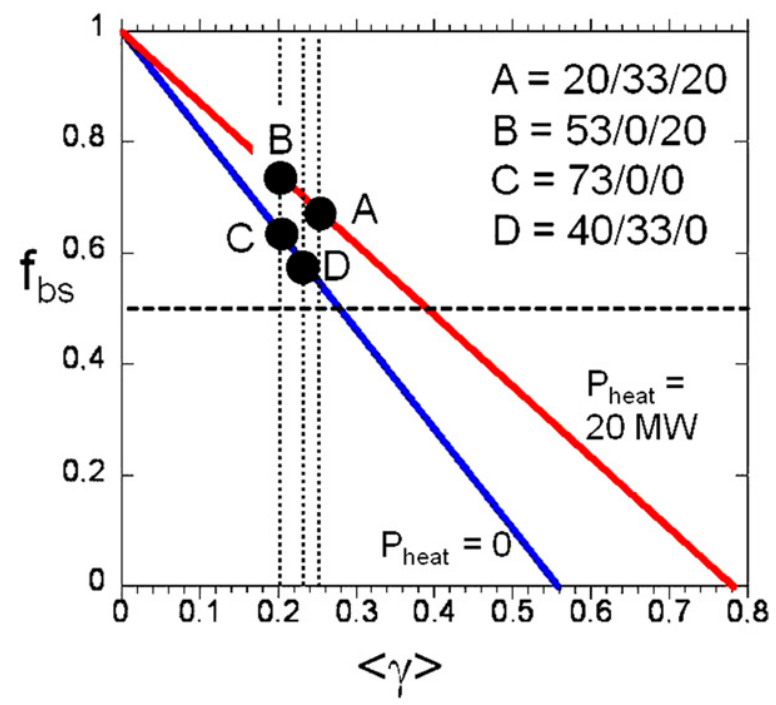

Figure 4. Plotted is the relation of $f_{\mathrm{bs}}$ with $\langle\gamma\rangle$ for $Q=5$ for $P_{\text {heat }}=20 \mathrm{MW}$ and $P_{\text {heat }}=0$. The locations of the four cases A-D (see table 2) are indicated by dotted lines.

The $\langle\gamma\rangle$ values of the heating scenarios A-D are indicated by vertical lines. For all cases, $f_{\mathrm{bs}}>0.5$ is required.

For $\operatorname{ECCD}\langle\gamma\rangle=0.22(0.16) \times 10^{20} \mathrm{~m}^{-2} \mathrm{~A} \mathrm{~W}^{-1}$ for $\rho=0(0.4)$ (see table 2$)$. The NBCD efficiency does not vary much with radius and is assumed to be 0.3 . ICH is used for central ion heating in scenarios $\mathrm{A}$ and $\mathrm{B}$.

With these $\langle\gamma\rangle$-values, the dependence of $Q$ versus $f_{\mathrm{bs}}$ of figure 5 is obtained for $P_{\text {fus }}=350 \mathrm{MW}$. Scenario D with the highest CD power reaches $Q \approx 4$ at $f_{\mathrm{bs}} \approx 0.5$ and needs $f_{\mathrm{bs}} \approx 0.6$ for $Q \approx 5$. Case B without NBCD reaches barely $Q=3$ at $f_{\mathrm{bs}} \approx 0.5$ and requires a much enhanced bootstrap fraction $(\approx 0.75)$ to reach $Q=5$.

Figure 6 shows the relation between $Q$ and the non-inductive current fraction $f_{\mathrm{ni}}=$ $f_{\mathrm{bs}}+I_{\mathrm{CD}} / I_{\mathrm{p}}$ for $P_{\text {heat }}=0$ and $P_{\text {heat }}=20 \mathrm{MW}$ (solid and dashed curves). The four reference cases A-D are plotted as solid points. Also variants of A and $\mathrm{C}$ were studied. These cases are results of steady-state solutions of iterative 1D transport modelling using the GLF23 transport model with existing DIII-D boundary information [27].

High $Q$ values are obtained with NBI or ECH, respectively. But only the scenarios with NBCD reach $f_{\text {ni }}$ values above 0.8 . Direct ion heating provides case B with a slightly higher 


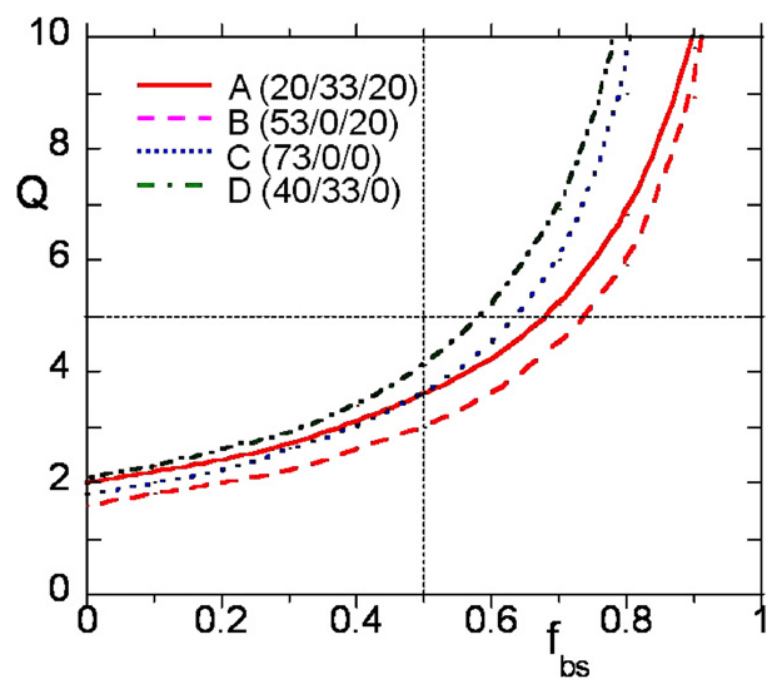

Figure 5. $Q$ versus $f_{\text {bs }}$ for the four cases A-D.

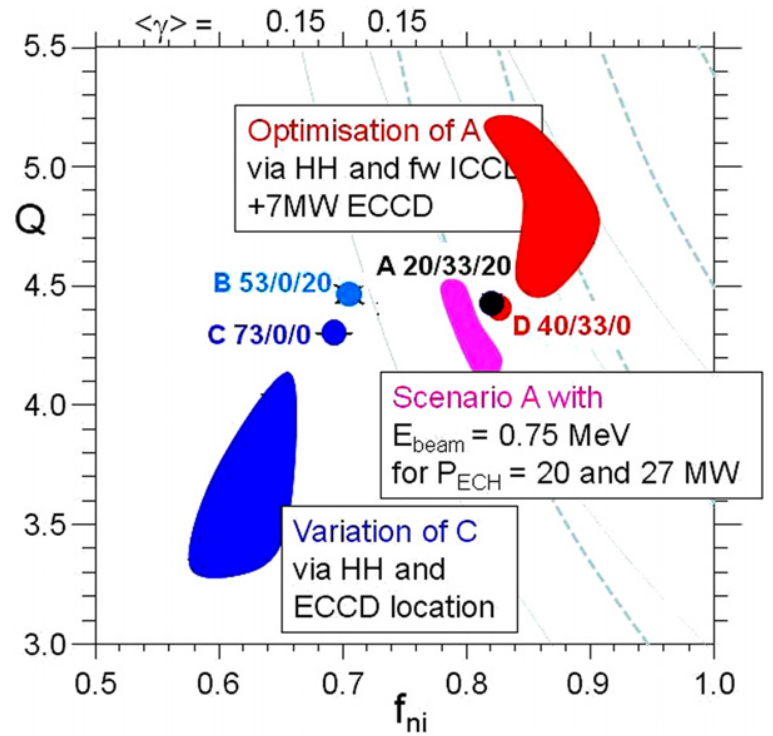

Figure 6. Operational space of $Q$ versus the non-inductive current fraction $f_{\text {ni }}$. Shown are the cases A-D and variants of A and C. For mix A, the consequences of reducing the beam energy to $0.75 \mathrm{MeV}$ was investigated. The curves are based on the simple relation with $f_{\mathrm{bs}}=0.5$ and variable $\langle\gamma\rangle$. The solid curves are with $P_{\text {heat }}=20 \mathrm{MW}$, the dashed ones with $P_{\text {heat }}=0 .\langle\gamma\rangle$ starts with 0.15 and increases in steps of 0.05 up to 0.3 for the two cases.

$Q$ than case C. Detailed simulations around the heating scenarios A and $\mathrm{C}$ were carried out. Using NBI and ECRH/ICH in various combinations, which always add up to $73 \mathrm{MW}$, high $f_{\text {ni }}$ and $\mathrm{Q}\left(f_{\text {ni }} \approx 0.8-0.9, Q \approx 4.5-5\right)$ can be obtained. With ECCD alone, different variants have been studied making use of the technical ECCD injection flexibility foreseen at ITER. $f_{\text {ni }}$ drops typically below 0.7 and about $2 \mathrm{MA}$ are missing, even when increasing the ECCD efficiency by $30 \%$. It is obvious that only with NBCD and optimized scenarios can one reach $f_{\text {ni }}$ values near 0.9 and $Q$ near 5 . 
The studies presented here are indicative only and not yet fully optimized. For example, better $Q$ values might be obtained with lower power, e.g. $P_{\mathrm{ECCD}}=53 \mathrm{MW}$ instead of $73 \mathrm{MW}$ for the steady-state phase.

Facing the difficulty with reaching truly steady-state conditions, it is worthwhile to also assess the heating scenarios, which maintain a small inductive current of the order of 1 MA [28] with $f_{\text {ni }}<1$. The ITER baseline heating scenario A has been studied with the GLF23 model and the fast transport code (FastTran), ONETWO and EFIT [29]. These runs have been performed keeping the total plasma current fixed at $9 \mathrm{MA}$. No further optimization of these scenarios was done. With an off-axis NBCD set-up, a reduction in $f_{\text {bs }}$ by 0.1 compensated by an ohmic contribution of $\approx 1 \mathrm{MA}\left(f_{\mathrm{ni}} \approx 0.9\right) Q \approx 4.5$ can be achieved. These discharges with finite ohmic current will allow ITER to reach the pulse length goal and they will play an important role in the development of steady-state scenarios and the preparation of the steady-state technology.

4.2.2. Sensitivity studies. In order to consolidate the conclusions of this paper on the proper heating mix of ITER a sequence of sensitivity studies has been carried out. They should also increase the awareness for the aspect that minor changes in plasma properties are capable of strongly enhancing the prospects of ITER. For modelling these special cases, reference is made to the two ITER scenario 4 discharges - type I with strongly reversed magnetic shear in the core and type II with weakly reversed shear (see figure 41 in [5]). These two cases represent the operational boundaries towards the strong ITB case with marginally stable $q$ at the plasma centre and the weak ITB case with $f_{\text {ni }}$ dropping below $100 \%$. These cases are modelled with $\mathrm{HH}=1.37$. The reference $q$ and $T_{\mathrm{e}}$ profiles are taken from [5]. If 1D transport modelling with alternative heating mixes reproduces these target profiles, steady-state performance can be expected.

High edge pedestal temperature. With the assumption of a high edge pedestal $\left(T_{\text {ped }}>7 \mathrm{keV}\right)$ leading to a high edge bootstrap current contribution, stationarity is obtained with the noninductive fraction, $f_{\mathrm{ni}}$, even in excess of $100 \% ; f_{\mathrm{bs}}=0.7, Q=5.3$ and $\beta_{\mathrm{N}}=3.1$ [30]. In the ONETWO code modelling off-axis NBI and ECCD were used for CD and ICH for central heating. No ITB is being developed.

High internal transport barrier. In this case weakly or strongly reversed $q$ profiles have to be tailored and sustained. $q(\rho)$ and $\chi(\rho)$ profile need to be consistent. It has been verified that there is the technical flexibility at ITER to correspondingly shape the $q$ profile by ECCD [31]. A weak shear case was explored in detail with $50 \mathrm{MW}$ of NBI and $20 \mathrm{MW}$ of ECCD. HH was assumed to be 1.37. $f_{\mathrm{bs}} \approx 0.5$ without a high temperature pedestal. At $I_{\mathrm{p}}=8.5 \mathrm{MA} Q=5$ was achieved steady state.

Case with ECCD alone. ECCD with $P_{\mathrm{CD}}=50 \mathrm{MW}$ and $70 \mathrm{MW}$ were studied. Without $\mathrm{NBCD}$, the total current drops down to $6 \mathrm{MA}$ causing a serious reduction in performance. $Q$ depends significantly on $I_{\mathrm{p}}\left(\sim I_{\mathrm{p}}^{3}\right)$. $50 \mathrm{MW}$ yields higher $Q$ values than $70 \mathrm{MW}$. This is because the increase in $I_{\mathrm{ECCD}}$ does not offset the increase in auxiliary power, due to the low average CD efficiency. $50 \mathrm{MW}$ ECCD yield $Q \approx 3$ with $\mathrm{HH}=1.37$ [32]. One way of compensation is by increasing the $\mathrm{HH}$ factor (and scaling thereby the effective $\chi(\rho)$ ). Again in the spirit of a sensitivity study $\mathrm{HH}$ has been varied between 1.3 and 1.78. Even for the highest $\mathrm{HH}$ factor the performance losses due to the lower total plasma current are not recovered. No steady-state scenarios are found with $f_{\text {ni }}>0.85$ and $Q$ near 5 with ECCD only or with ECCD and ICCD. 
Doubling the core ECCD efficiency. This case represents a further sensitivity study where $\gamma$ was increased by a factor of 2 in the core where temperatures are highest and trapped particle fraction smallest. At the edge, there is no enhancement because the linear predictions are considered to be relevant. Even with this optimistic assumption and $\mathrm{HH}=1.78$, $I_{\mathrm{ECCD}}<3 \mathrm{MA}, I_{\mathrm{p}} \approx 7 \mathrm{MA}$ and $Q$ just below 5 are obtained. The strategy to maximize the driven current while $q$ is kept above 1 leads to a current profile clearly too peaked and not compatible with $\mathrm{HH}=1.78$.

LH and NBCD. Steady-state profiles have been obtained with weak and strongly reversed shear with $33 \mathrm{MW}$ of NBI and $34 \mathrm{MW}$ of LHCD at an HH factor of 1.37 . $Q=5$ at $I_{\mathrm{p}}=9 \mathrm{MA}$ is achieved with profiles similar to the ITER reference ones. The current density profile for LHCD is taken from [33]. However, the LHCD efficiency of $0.3 \mathrm{~A} \mathrm{~W}^{-1} 10^{20} \mathrm{~m}^{-2}$ is now considered as too optimistic due to a lack of directivity [34]. Furthermore, it is unlikely that $30 \mathrm{MW}$ of LH power could be launched from a single ITER port. Therefore, an injected LH power limited to $20 \mathrm{MW}$, with a $70 \%$ directivity should be considered as the reference operation value.

$L H C D, I C R H$ and ECCD. For this RF-only case [35] current density maxima of $j_{\mathrm{bs}}$ and $j_{\mathrm{ECCD}}$ coincide at $\rho=0.45$. Here an ITB develops which is triggered and locked by ECCD. The LHCD power deposition is located at $\rho=0.7$ and the $\mathrm{CD}$ obtained $(\approx 0.6 \mathrm{MA})$ contributes to the total non-inductive current fraction $\left(f_{\mathrm{ni}} \approx 0.97\right)$. ICH provides central heating. Currents driven inside the ITB have been found to lead to shrinking and final collapse of the ITB itself [28]. This is the well-known problem of current alignment, i.e. an incompatibility of the profiles of non-inductively driven and bootstrap current can prevent steady-state profiles to be sustained [36]. The details depend, however, on the transport model relating shear and $\chi$. With this CD scheme, the $q$ profile obtained is stable for $1000 \mathrm{~s}$ at $I_{\mathrm{p}}=8 \mathrm{MA}$, with $q_{0} \approx 6$ and $q_{\min }>2$. $Q=6.5$ is obtained. This scenario is, however, rather demanding in terms of MHD. Owing to the strongly negative shear combined with large pressure gradients, theses plasmas are prone to resistive interchange modes [35].

\section{Considerations regarding $\mathrm{CD}$ technology}

\subsection{NBI}

High-power NNBI at $1 \mathrm{MeV}$ energy requires extensive R\&D. Such ion sources are not yet available, the $R \& D$ costs and the system costs are high and the time schedule is challenging. Because of the considerable risks the use of $0.85 \mathrm{MeV}$ instead of $1 \mathrm{MeV}$ beam energy has been considered as risk mitigation. The modelling-done with different code packagesindicates that such a reduction in the beam energy reduces the CD efficiency by less than $20 \%$. This is acceptable if the overall power is maintained at the $33 \mathrm{MW}$ level. This can be achieved by operating at increased current density from the ion source, by reducing the losses in the injector and the NB duct, or a combination of both. At a beamlet divergence $\approx 5 \mathrm{mrad}$, as already operated at the prototype accelerator at JAEA [37], a beam energy of only $\approx 0.85 \mathrm{MeV}$ would provide $16.5 \mathrm{MW}$ per ITER injector. Improvements in current density up to $30 \%$ can realistically be expected, but the co-extracted electron current must be controlled. The reduction in beam energy would not affect the goals of scenario 2; the decrease in the $\mathrm{CD}$ efficiency for scenario 4 (see figure 6 for $0.75 \mathrm{MeV}$ beam energy) can be compensated with ECCD or-more speculatively-via a higher bootstrap current arising from the increased torque at lower beam energy improving confinement. 
Considering the substantial effort required a critical issue is whether NNBI can also drive the current required for DEMO [38]. DEMO can be heated without NBI and steady-state CD can be achieved with ECCD. However, the increased CD power required in this case necessitates an enlargement of the unit size to deliver the same output. This leads to an increase of perhaps $25 \%$ both in the capital and the electricity costs, the cost driving factor being the recirculating power for CD. Whereas a steady-state DEMO with NBI needs a CD power of about $200 \mathrm{MW}$, it will need twice that power with ECCD. The R\&D for the NBI system should therefore remain targeted for $1 \mathrm{MeV}$ beam energy, which will—considering also DEMO_reduce the overall development costs. It seems that a decision against NBI on ITER risks removing an important option for steady-state operation of DEMO.

\section{2. $E C H$}

There is sufficient know-how and capacity across the ITER partners to ensure timely delivery of gyrotrons with at least $1 \mathrm{MW}$ unit output. The overall R\&D risks are low because ITER-like systems are well advanced for W7-X [39] and the gyrotron R\&D for ITER has already met the specifications. The use of diplexers [40] and/or gyrotrons with higher power ( $>1 \mathrm{MW}$ ) would allow the full exploitation of the foreseen transmission and launching systems thus reducing the space demand, number of transmission lines and system complexity in close neighbourhood of the device, and therefore also substantially reducing the marginal cost of a power enhancement. A future enhancement in ECH power up to a total of $40 \mathrm{MW}$ is recommended (using the capability of the existing launchers) to increase the operating margins across the scenarios. This would also mitigate possible consequences of the technical development risks of NNBI or the coupling risks of ICH. Only ECH seems to be sufficiently technically mature at present to justify a power increase.

\section{3. $\mathrm{ICH}$}

$\mathrm{ICH}$ is the heating method with the lowest costs. But ICH is different from the other heating mechanisms because prior knowledge of plasma properties (density in the outer scrape-off layer) is necessary for precise coupling predictions. Experience with the operation of the ITER-like ICH antenna on JET and the consequences of the follow-up analysis of the observed low coupling under $\mathrm{H}$-mode edge conditions [41] will enter the ITER antenna design. The installation of two antennae (for a total of $20 \mathrm{MW}$ coupled power) in the early hydrogen and deuterium phases of the project will improve the prospects for the timely availability of sufficient additional heating power.

\section{4. $L H$}

LH has not been explored in any detail here but this study has clearly shown a demand for off-axis CD with high efficiency. This is the main feature of LH. LH does not appear to be mandatory but would facilitate the achievement of scenario 4-like discharges or-more generally - the prospects of steady-state operation. The ITER requirement to be able to accommodate up to $40 \mathrm{MW}$ of $\mathrm{LH}$ in two ports, but not yet to precede with design and procurement, is supported here. Because of the criticality of steady-state operation, LH should be reconsidered after operational experience has been gained and the need for off-axis CD has been clarified. This will only be possible, however, with timely implementation of the necessary R\&D. 


\section{Conclusions and summary}

The presently foreseen heating mix A seems to best ensure the accessibility to the full range of ITER operation scenarios whilst providing the flexibility required for an experimental device. The heating mix seems to be uncritical for scenario 2 . ICH and a peaked density profile would support the development of $Q=10$. Critical seems to be the edge temperature pedestal and the edge pressure gradient. H-mode versions with improved core confinement-not considered in this study-could overcome possible limitations of the baseline scenario.

Scenario 4 clearly needs in situ development and will be part of the experimental programme of ITER. For its success, a flexible heating system is necessary. Secondary characteristics of the various methods can be utilized as actuators to induce specific plasma responses. Owing to the limitations in the $\mathrm{CD}$ efficiencies of the presently foreseen systems, LH should be considered for a later experimental stage.

NBI seems to be indispensable due to the high off-axis CD efficiency. The technical development risks with beam energies up to $1 \mathrm{MeV}$ are high, however. A lower beam energy target of $0.85 \mathrm{MeV}$ would be acceptable for ITER. For the DEMO needs the NNBI R\&D should, however, target for $1 \mathrm{MeV}$ beams. ECH is technically mature in the present frequency range. Its off-axis CD efficiency is too low, however, to serve as the exclusive CD method.

ITER will be limited to $Q \approx 2-3$ if the H-mode is not achieved. The DT power threshold for ITER plateau conditions is about $70 \mathrm{MW}(40 \mathrm{MW})$ for scenario 2 (scenario 4). Furthermore, high-confinement $\mathrm{H}$-modes will require a power typically $20 \%$ above threshold [42] depending, however, on the ELM type which develops [43]. This compares with $73 \mathrm{MW}$ of external heating and $\approx 40 \mathrm{MW}$ of $P_{\alpha}$ in the L-mode of scenario 2 settings just prior to the H-transition. The power margin for scenario 2 is critical and is much smaller than for any of the experiments, which prepared the data basis for ITER. Hydrogen or helium plasmas foreseen for the early phases will have even higher H-mode thresholds. It is therefore suggested to increase this power margin at ITER by additional $20 \mathrm{MW}$ of ECH.

\section{Acknowledgments}

The authors would like to acknowledge the contributions from F4E and ITER IO to the original study and to this paper. This publication is dedicated to the authors' good friend and colleague, the late Arturo Tanga, who throughout the study updated AG-2 with the progress of ITER heating systems.

\section{References}

[1] Wilhelm R 1998 Plasma Phys. Control. Fusion 40 A1

[2] 2007 Progress in the ITER Physics Basis: chapter 1 Nucl. Fusion 47 S1

[3] ITER Physics Teams 1999 Nucl. Fusion 392541

[4] Sips A C et al 22nd IAEA Fusion Energy Conf. paper IAEA-CN-165/IT/2-2

[5] Gormezano C et al 2007 Progress in the ITER Physics Basis: chapter 6 Nucl. Fusion 47 S285

[6] Waltz R W et al 1997 Phys. Plasmas 42482 http://w3.pppl.gov/NTCC/GLF

[7] Doyle E J et al 2007 Progress in the ITER Physics Basis: chapter 2, section 3 Nucl. Fusion 47 S18

[8] Budny R V 2009 Nucl. Fusion 49085008

[9] Angioni C et al 2003 Phys. Rev. Lett. 90205003

[10] Angioni C et al 2009 Plasma Phys. Control. Fusion 51124017

[11] Weisen H et al 2001 Nucl. Fusion 411227

Gohill P et al 2003 Plasma Phys. Control. Fusion 45601

Ryter F et al 2001 Nucl. Fusion 41537

Takenaga H, et al 2003 Nucl. Fusion 431235 
[12] Erckmann V et al 1993 Proc. 20th EPS Conf. on Controlled Fusion and Plasma Physics (Lisboa, Portugal) vol 17C, Part I, pp 345-8

[13] Prater R et al 2008 Nucl. Fusion 48035006

[14] Harvey R W and McCoy M G 1993 Proc. IAEA Technical Committee Meeting (Montreal, 1992)(Vienna: IAEA) p 498

[15] Marushchenko N et al 2008 Nucl. Fusion 48054002 Marushchenko N et al 2009 Nucl. Fusion 49129801

[16] Zvonkov A V et al 1998 Plasma Phys. Rep. 24389 Farina D 2007 Fusion Sci. Technol. 52154

[17] Poli E, Pereverzev G V and Peeters A G 1999 Phys. Plasmas 65

[18] Ramponi G et al 2007 Fusion Eng. Des. 82454

[19] Oikawa T et al 2001 Nucl. Fusion 411575

[20] Tuszewiski M and Roubin J P 1988 Nucl. Fusion 28499

[21] Pankin A et al 1981 Comput. Phys. Commun. 4361

[22] Heidbrink W W et al 2009 Phys. Rev. Lett. 103175001

[23] Cenacchi G and Taroni A 1986 Proc. 8th Comput. Physics, Computing in Plasma Physics (Eibsee, Germany 1986) (Petit-Lancy: European Physical Society) vol 10D, p 57

[24] Mikhailovskii A B et al 1997 Plasma Phys. Rep. 23

[25] Snyder P B et al 2002 Phys. Plasmas 92037

[26] Kessel C E et al 2009 Nucl. Fusion 49085034

[27] Park J M et al 2010 IAEA Fusion Energy Conf. Nucl. Fusion to be submitted

[28] Budny R V 2010 Phys. Plasmas 17042506

[29] St John H et al 1993 Proc. Conf. on Controlled Fusion and Plasma Physics (Lisboa, Portugal, 1993) (Geneva: European Physical Society) vol I, p 99

[30] Giruzzi G et al 2008 21st IAEA Fusion Energy Conf. (Chengdu) paper IT/P6-4

[31] Zucca C 2009 PhD Thesis EPFL, no 4360 http://library.epfl.ch/en/theses/?nr=4360

[32] Sauter O and Zucca C 2009 On the role of heating and current drive sources on the scenario 4 for ITER: preliminary results with EC only report

[33] Bonoli P T et al 2006 Proc. 21st Int. Conf. on Fusion Energy 2006 (Chengdu, China, 2006) (Vienna: IAEA) CD-ROM file IT/P1-2

[34] Hoang G T et al 2009 Nucl. Fusion 49075001

[35] Garcia J et al 2008 Phys. Rev. Lett. 100255004

[36] Garcia J and Giruzzi G 2010 Phys. Rev. Lett. 104205003

[37] Taniguchi M et al 2008 Rev Sci. Instrum. $7902 \mathrm{C} 110$

[38] Ward D 2010 Plasma Phys. Control. Fusion 52124033 (this issue)

[39] Erckmann V et al 2007 Fusion Sci. Technol. 52291

[40] Erckmann V et al 2009 Fusion Sci. Technol. 5523

[41] Nightingale M P S et al 2009 Radio frequency power in plasmas AIP Conf. Proc. 1187213

[42] Satori R et al 2004 Plasma Phys. Control. Fusion 46723

[43] Ryter F et al 2008 J. Phys.: Conf. Ser. 123012035 